Article

\title{
Association between Occupational Noise and Vibration Exposure and Insomnia among Workers in Korea
}

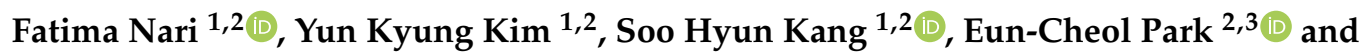 \\ Sung-In Jang ${ }^{2,3, *}$ \\ 1 Department of Public Health, Graduate School, Yonsei University, Seoul 03722, Korea; fatima@yuhs.ac (F.N.); \\ hykh51128@yuhs.ac (Y.K.K.); kshyun@yuhs.ac (S.H.K.) \\ 2 Institute of Health Services Research, Yonsei University, Seoul 03722, Korea; ecpark@yuhs.ac \\ 3 Department of Preventive Medicine, Yonsei University College of Medicine, 50 Yonsei-ro, Seodaemun-gu, \\ Seoul 03722, Korea \\ * Correspondence: jangsi@yuhs.ac; Tel.: +82-2-2228-1862; Fax: +82-2-392-8133
}

Received: 20 February 2020; Accepted: 21 April 2020; Published: 22 April 2020

\begin{abstract}
Background: The effect of noise and vibration exposure on disturbed sleep has been investigated in the past. However, this study was carried out to investigate the relationship between workplace noise and vibration exposure with insomnia amongst representative Korean workers, both simultaneously and separately. Methods: Our research analyzed an overall population of 30,837 workers aged 15 years or older using data derived from the 5th Korean Working Conditions Survey (KWCS) conducted in 2017. Chi-squared tests and logistic regression were performed to investigate baseline characteristics and to quantify the association between workplace exposure to noise and vibration with insomnia. Relative excess risk due to interaction (RERI), attributable proportion (AP), and synergy index (S) were calculated to measure interactions between simultaneous noise and vibration exposure with insomnia. Results: The prevalence of those who reported insomnia was $18.3 \%$ of the general population. Among men and women, insomnia in those who were exposed to noise only was $13.9 \%$ and $18.3 \%$, respectively, and in those who were exposed to vibration only, it was $23.9 \%$ in males and $26.4 \%$ in females. Insomnia in those who were exposed to both noise and vibration simultaneously was $20.5 \%$ and $41.2 \%$ in men and women, respectively. The odds ratio (OR) of insomnia due to noise exposure was 1.10 and 1.07 in men and women, respectively. OR of vibration exposure was 1.84 in men and 1.58 in women. For noise plus vibration exposure OR was 1.83 in men and 3.14 in female workers, where the synergistic effect of noise and vibration exposure could be seen. The association between the varying degree of simultaneous noise plus vibration exposure with insomnia showed a dose-response relationship. The interaction measures showed a synergistic effect of simultaneous exposure in women but not in men. Conclusion: Our study revealed an association between occupational noise and vibration exposure and insomnia, both individually and simultaneously. Additional studies and research are required to further comprehend this relationship.
\end{abstract}

Keywords: sleep disorders; insomnia; disorders of initiating and maintaining sleep; occupational noise exposure; occupational vibration exposure; occupational health

\section{Introduction}

Over the past few decades, an emphasis has been placed on investigating the effects of different occupational risk hazards on the safety and wellbeing of workers. Exposure to environmental stressors such as noise and vibration and their detrimental effects on health and diseases have been explored frequently in other studies. Some of these effects include, but are not limited to, physiological conditions 
such as hearing loss [1], cardiac and vascular problems [2,3], and elevated blood pressure [4,5], as well as nervous system-related disorders such as stress, annoyance [6], headache/eye strain [7], fatigue [8], and perhaps the most prominent nervous system issue-insomnia/sleep disturbance [9-11].

Sleep is essential for regeneration of the body [12], and therefore, poor quality of sleep or disturbed sleep can be of significance for immediate and long-term health [13]. Insomnia may be of qualitative or quantitative nature and is characterized by difficulty of falling asleep and frequent awakenings leading to unsatisfying or unrefreshing sleep [11,14]. Disturbed sleep can result in consequential effects such as daytime sleepiness [15], fatigue, and reduced mental and cognitive function [16,17]. Negative outcomes of insufficient sleep in the workplace include increased workplace aggression [18], decreased work productivity, absenteeism, and a rise in the number of occupational accidents and injuries $[19,20]$.

The adverse effects of noise on sleep have been thoroughly researched, and there are existing guidelines regarding noise exposure consequences [21], but the mechanism of how vibration influences sleep is still unclear. Even more lacking is knowledge relating to the synergistic effect of combined noise and vibration exposure on insomnia. Investigations regarding occupational noise and vibration exposures can be quite challenging due to the fact that extra-occupational sources of noise and vibration are quite numerous [22-24]. Therefore, it is difficult to identify risks associated with those two factors.

Our objective in this study was to investigate the association between noise and vibration exposure and insomnia both individually and together. This set it apart from prior studies that concentrated on the relationship between insomnia and each of the two risk factors separately. In addition, we aimed to find out whether the combined effect of occupational noise and vibration exposures was synergistic and to compare the two factors and determine which one has a larger influence on insomnia.

\section{Results}

The general characteristics of the 30,827 study participants are summarized in Table 1 by gender (14,383 men and 16,444 women). Among the study population, the total number of those who reported the presence of insomnia was $18.3 \%$, and men and women with insomnia who reported being exposed to noise only were $13.9 \%$ and $18.3 \%$, respectively. Additionally, insomnia in those who were exposed to vibration only was $23.9 \%$ in males and $26.4 \%$ in females. Those who were exposed to both noise and vibration at the same time and reported insomnia were $20.5 \%$ and $41.2 \%$ in men and women, respectively. 
Table 1. General characteristics of study population.

\begin{tabular}{|c|c|c|c|c|c|c|c|c|c|c|c|c|c|c|}
\hline \multirow{4}{*}{ Variables } & \multicolumn{14}{|c|}{ Insomnia } \\
\hline & \multicolumn{6}{|c|}{ Male $(N=14,383)$} & \multirow{3}{*}{$p$-Value } & \multicolumn{6}{|c|}{ Female $(N=16,444)$} & \multirow{3}{*}{$p$-Value } \\
\hline & \multicolumn{2}{|c|}{$N=14,383$} & \multicolumn{2}{|c|}{ Yes } & \multicolumn{2}{|c|}{ No } & & \multicolumn{2}{|c|}{$N=16,444$} & \multicolumn{2}{|c|}{ Yes } & \multicolumn{2}{|c|}{ No } & \\
\hline & $N$ & $\%$ & $N$ & $\%$ & $N$ & $\%$ & & $N$ & $\%$ & $N$ & $\%$ & $N$ & $\%$ & \\
\hline Noise and vibration exposure & & & & & & & $<0.0001$ & & & & & & & $<0.0001$ \\
\hline No Exposure & 8621 & 59.9 & 1274 & 14.8 & 7347 & 85.2 & & 13,028 & 79.2 & 2267 & 17.4 & 10,761 & 82.6 & \\
\hline Noise Exposure & 1816 & 12.6 & 252 & 13.9 & 1564 & 86.1 & & 1191 & 7.2 & 218 & 18.3 & 973 & 81.7 & \\
\hline Vibration Exposure & 833 & 5.8 & 199 & 23.9 & 634 & 76.1 & & 889 & 5.4 & 235 & 26.4 & 654 & 73.6 & \\
\hline Noise plus Vibration exposure & 3113 & 21.6 & 637 & 20.5 & 2476 & 79.5 & & 1336 & 8.1 & 551 & 41.2 & 785 & 58.8 & \\
\hline Age & & & & & & & $<0.0001$ & & & & & & & $<0.0001$ \\
\hline$\leq 29$ & 1338 & 9.3 & 159 & 11.9 & 1179 & 88.1 & & 1235 & 7.5 & 177 & 14.3 & 1058 & 85.7 & \\
\hline $30-39$ & 2256 & 15.7 & 332 & 14.7 & 1924 & 85.3 & & 1961 & 11.9 & 311 & 15.9 & 1650 & 84.1 & \\
\hline $40-49$ & 2979 & 20.7 & 439 & 14.7 & 2540 & 85.3 & & 3576 & 21.7 & 615 & 17.2 & 2961 & 82.8 & \\
\hline 50-59 & 3621 & 25.2 & 633 & 17.5 & 2988 & 82.5 & & 4970 & 30.2 & 1006 & 20.2 & 3964 & 79.8 & \\
\hline $60 \leq$ & 4189 & 29.1 & 799 & 19.1 & 3390 & 80.9 & & 4702 & 28.6 & 1162 & 24.7 & 3540 & 75.3 & \\
\hline Education & & & & & & & 0.0001 & & & & & & & $<0.0001$ \\
\hline Middle school degree & 2741 & 19.1 & 515 & 18.8 & 2226 & 81.2 & & 4386 & 26.7 & 1088 & 24.8 & 3298 & 75.2 & \\
\hline High School degree & 6189 & 43.0 & 1010 & 16.3 & 5179 & 83.7 & & 7132 & 43.4 & 1382 & 19.4 & 5750 & 80.6 & \\
\hline University degree or higher & 5453 & 37.9 & 837 & 15.3 & 4616 & 84.7 & & 4926 & 30.0 & 801 & 16.3 & 4125 & 83.7 & \\
\hline Income ${ }^{a}$ & & & & & & & 0.0003 & & & & & & & 0.1309 \\
\hline Q1 & 2182 & 15.2 & 406 & 18.6 & 1776 & 81.4 & & 5540 & 33.7 & 1138 & 20.5 & 4402 & 79.5 & \\
\hline Q2 & 3751 & 26.1 & 628 & 16.7 & 3123 & 83.3 & & 6277 & 38.2 & 1244 & 19.8 & 5033 & 80.2 & \\
\hline Q3 & 4248 & 29.5 & 698 & 16.4 & 3550 & 83.6 & & 3032 & 18.4 & 578 & 19.1 & 2454 & 80.9 & \\
\hline Q4 & 4202 & 29.2 & 630 & 15.0 & 3572 & 85.0 & & 1595 & 9.7 & 311 & 19.5 & 1284 & 80.5 & \\
\hline Depression & & & & & & & $<0.0001$ & & & & & & & $<0.0001$ \\
\hline Yes & 372 & 2.6 & 156 & 41.9 & 216 & 58.1 & & 558 & 3.4 & 260 & 46.6 & 298 & 53.4 & \\
\hline No & 14,011 & 97.4 & 2206 & 15.7 & 11,805 & 84.3 & & 15,886 & 96.6 & 3011 & 19.0 & 12,875 & 81.0 & \\
\hline Fatigue & & & & & & & $<0.0001$ & & & & & & & $<0.0001$ \\
\hline Yes & 3962 & 27.5 & 806 & 20.3 & 3156 & 79.7 & & 4603 & 28.0 & 1108 & 24.1 & 3495 & 75.9 & \\
\hline No & 10,421 & 72.5 & 1556 & 14.9 & 8865 & 85.1 & & 11,841 & 72.0 & 2163 & 18.3 & 9678 & 81.7 & \\
\hline Hearing Problems & $10, \pm \angle 1$ & & & & & & $<0.0001$ & & & & & & & $<0.0001$ \\
\hline Yes & 262 & 1.8 & 80 & 30.5 & 182 & 69.5 & & 230 & 1.4 & 106 & 46.1 & 124 & 53.9 & \\
\hline No & 14,121 & 98.2 & 2282 & 16.2 & 11,839 & 83.8 & & 16,214 & 98.6 & 3165 & 19.5 & 13,049 & 80.5 & \\
\hline Headache/Eye strain & & & & & & & $<0.0001$ & & & & & & & $<0.0001$ \\
\hline Yes & 2002 & 13.9 & 475 & 23.7 & 1527 & 76.3 & & 2361 & 14.4 & 667 & 28.3 & 1694 & 71.7 & \\
\hline No & 12,381 & 86.1 & 1887 & 15.2 & 10,494 & 84.8 & & 14,083 & 85.6 & 2604 & 18.5 & 11,479 & 81.5 & \\
\hline Subjective Health Condition & & & & & & & $<0.0001$ & & & & & & & $<0.0001$ \\
\hline Good & 9538 & 66.3 & 1,305 & 13.7 & 8233 & 86.3 & & 9843 & 59.9 & 1570 & 16.0 & 8273 & 84.0 & \\
\hline Normal & 4208 & 29.3 & 856 & 20.3 & 3352 & 79.7 & & 5325 & 32.4 & 1215 & 22.8 & 4110 & 77.2 & \\
\hline Bad & 637 & 4.4 & 201 & 31.6 & 436 & 68.4 & & 1276 & 7.8 & 486 & 38.1 & 790 & 61.9 & \\
\hline
\end{tabular}


Table 1. Cont

\begin{tabular}{|c|c|c|c|c|c|c|c|c|c|c|c|c|c|c|}
\hline \multirow{4}{*}{ Variables } & \multicolumn{14}{|c|}{ Insomnia } \\
\hline & \multicolumn{6}{|c|}{ Male $(N=14,383)$} & \multirow{3}{*}{$p$-Value } & \multicolumn{6}{|c|}{ Female $(N=16,444)$} & \multirow{3}{*}{$p$-Value } \\
\hline & \multicolumn{2}{|c|}{$N=14,383$} & \multicolumn{2}{|c|}{ Yes } & \multicolumn{2}{|c|}{ No } & & \multicolumn{2}{|c|}{$N=16,444$} & \multicolumn{2}{|c|}{ Yes } & \multicolumn{2}{|c|}{ No } & \\
\hline & $N$ & $\%$ & $N$ & $\%$ & $N$ & $\%$ & & $N$ & $\%$ & $N$ & $\%$ & $N$ & $\%$ & \\
\hline Physical Activity in Leisure Time & & & & & & & 0.4184 & & & & & & & 0.9415 \\
\hline Everyday & 326 & 2.3 & 64 & 19.6 & 262 & 80.4 & & 268 & 1.6 & 65 & 24.3 & 203 & 75.7 & \\
\hline Several times per week & 1658 & 11.5 & 315 & 19.0 & 1343 & 81.0 & & 1488 & 9.0 & 373 & 25.1 & 1115 & 74.9 & \\
\hline Several times per month & 3446 & 24.0 & 499 & 14.5 & 2947 & 85.5 & & 3151 & 19.2 & 560 & 17.8 & 2591 & 82.2 & \\
\hline Rarely & 3939 & 27.4 & 571 & 14.5 & 3368 & 85.5 & & 4219 & 25.7 & 691 & 16.4 & 3528 & 83.6 & \\
\hline Never & 5014 & 34.9 & 913 & 18.2 & 4101 & 81.8 & & 7318 & 44.5 & 1582 & 21.6 & 5736 & 78.4 & \\
\hline Use of PPE $b$ & & & & & & & $<0.0001$ & & & & & & & 0.1281 \\
\hline Yes & 4689 & 32.6 & 595 & 12.7 & 4094 & 87.3 & & 3246 & 19.7 & 582 & 17.9 & 2664 & 82.1 & \\
\hline No & 704 & 4.9 & 136 & 19.3 & 568 & 80.7 & & 608 & 3.7 & 186 & 30.6 & 422 & 69.4 & \\
\hline Not applicable & 8990 & 62.5 & 1631 & 18.1 & 7359 & 81.9 & & 12,590 & 76.6 & 2503 & 19.9 & 10,087 & 80.1 & \\
\hline Job Satisfaction & & & & & & & $<0.0001$ & & & & & & & $<0.0001$ \\
\hline Yes & 10,253 & 71.3 & 1418 & 13.8 & 8835 & 86.2 & & 4167 & 25.3 & 1255 & 30.1 & 2912 & 69.9 & \\
\hline No & 4130 & 28.7 & 944 & 22.9 & 3186 & 77.1 & & 12,277 & 74.7 & 2016 & 16.4 & 10,261 & 83.6 & \\
\hline Work and life balance & & & & & & & $<0.0001$ & & & & & & & $<0.0001$ \\
\hline Yes & 9490 & 66.0 & 1432 & 15.1 & 8058 & 84.9 & & 11,387 & 69.2 & 2158 & 19.0 & 9229 & 81.0 & \\
\hline No & 4893 & 34.0 & 930 & 19.0 & 3963 & 81.0 & & 4987 & 30.3 & 1113 & 22.3 & 3874 & 77.7 & \\
\hline Work duration & & & & & & & 0.3461 & & & & & & & $<0.0001$ \\
\hline$\leq 5$ years & 4887 & 34.0 & 787 & 16.1 & 4100 & 83.9 & & 7063 & 43.0 & 1234 & 17.5 & 5829 & 82.5 & \\
\hline 5-10 years & 4367 & 30.4 & 715 & 16.4 & 3652 & 83.6 & & 4922 & 29.9 & 976 & 19.8 & 3946 & 80.2 & \\
\hline$\geq 11$ years & 5130 & 35.7 & 861 & 16.8 & 4269 & 83.2 & & 4459 & 27.1 & 1061 & 23.8 & 3398 & 76.2 & \\
\hline Working hours/week & & & & & & & 0.1910 & & & & & & & $<0.0001$ \\
\hline$\leq 40 \mathrm{~h}$ & 2412 & 16.8 & 463 & 19.2 & 1949 & 80.8 & & 5143 & 31.3 & 1122 & 21.8 & 4021 & 78.2 & \\
\hline $41-50 \mathrm{~h}$ & 6042 & 42.0 & 933 & 15.4 & 5109 & 84.6 & & 5704 & 34.7 & 1131 & 19.8 & 4573 & 80.2 & \\
\hline $51-60 \mathrm{~h}$ & 3913 & 27.2 & 614 & 15.7 & 3299 & 84.3 & & 4000 & 24.3 & 704 & 17.6 & 3296 & 82.4 & \\
\hline$\geq 61 \mathrm{~h}$ & 2016 & 14.0 & 352 & 17.5 & 1664 & 82.5 & & 1597 & 9.7 & 314 & 19.7 & 1283 & 80.3 & \\
\hline Job Collar $^{\mathrm{c}}$ & & & & & & & 0.0919 & & & & & & & 0.4592 \\
\hline White & 2518 & 17.5 & 365 & 14.5 & 2153 & 85.5 & & 2895 & 17.6 & 456 & 15.8 & 2439 & 84.2 & \\
\hline Blue & 7902 & 54.9 & 1345 & 17.0 & 6557 & 83.0 & & 4872 & 29.6 & 1180 & 24.2 & 3692 & 75.8 & \\
\hline Pink & 3963 & 27.6 & 652 & 16.5 & 3311 & 83.5 & & 8677 & 52.8 & 1635 & 18.8 & 7042 & 81.2 & \\
\hline Shift Work & & & & & & & 0.0204 & & & & & & & 0.5379 \\
\hline Yes & 1478 & 10.3 & 274 & 18.5 & 1204 & 81.5 & & 1151 & 7.0 & 237 & 20.6 & 914 & 79.4 & \\
\hline No & 12,905 & 89.7 & 2088 & 16.2 & 10,817 & 83.8 & & 15,293 & 93.0 & 3034 & 19.8 & 12,259 & 80.2 & \\
\hline Flexible break time & & & & & & & 0.0706 & & & & & & & $<0.0001$ \\
\hline Yes & 11,140 & 77.5 & 1863 & 16.7 & 9277 & 83.3 & & 12,255 & 74.5 & 2599 & 21.2 & 9656 & 78.8 & \\
\hline No & 3243 & 22.5 & 499 & 15.4 & 2744 & 84.6 & & 4189 & 25.5 & 672 & 16.0 & 3517 & 84.0 & \\
\hline Size of Business & & & & & & & 0.0038 & & & & & & & 0.0671 \\
\hline 1-9 people & 10,416 & 72.4 & 1767 & 17.0 & 8649 & 83.0 & & 13,199 & 80.3 & 2668 & 20.2 & 10,531 & 79.8 & \\
\hline 10-249 people & 3467 & 24.1 & 525 & 15.1 & 2942 & 84.9 & & 2976 & 18.1 & 549 & 18.4 & 2427 & 81.6 & \\
\hline$\geq 250$ people & 500 & 3.5 & 70 & 14.0 & 430 & 86.0 & & 269 & 1.6 & 54 & 20.1 & 215 & 79.9 & \\
\hline
\end{tabular}

a income level per month, which was divided into four quartiles $(<150,000 ;<250,000 ;<350,000, \geq 350,000)$; ${ }^{\mathrm{b}}$ Personal Protective Equipment; ${ }^{\mathrm{T}}$ Job collar types classified according the Korean Standard Occupational Classification. 
The results of the association between noise and vibration exposure and insomnia are shown in Table 2. The odds ratio [OR] of insomnia among male and female workers who were exposed to noise and/or vibration compared to workers who were not exposed is shown as follows: noise exposure: in men 1.10 (95\% CI 0.94-1.28) and in women 1.07 (95\% CI 0.91-1.26); vibration exposure: in men 1.84 (95\% CI 1.54-2.19) and in women 1.58 (95\% CI 1.34-1.86); noise plus vibration exposure: 1.83 (95\% CI 1.61-2.07) and 3.14 (95\% CI 2.76-3.57) in men and women, respectively.

Table 2. Association of Noise \& Vibration exposure with Insomnia.

\begin{tabular}{|c|c|c|c|c|}
\hline \multirow{3}{*}{ Variables } & \multicolumn{4}{|c|}{ Insomnia } \\
\hline & \multicolumn{2}{|c|}{ Male } & \multicolumn{2}{|c|}{ Female } \\
\hline & Adjusted OR & $95 \%$ CI & Adjusted OR & $95 \%$ CI \\
\hline \multicolumn{5}{|l|}{ Noise and vibration exposure } \\
\hline No Exposure & 1 & - & 1 & - \\
\hline Noise Exposure & 1.1 & $(0.94-1.28)$ & 1.07 & $(0.91-1.26)$ \\
\hline Vibration Exposure & 1.84 & $(1.54-2.19)$ & 1.58 & $(1.34-1.86)$ \\
\hline Noise plus Vibration exposure & 1.83 & $(1.61-2.07)$ & 3.14 & $(2.76-3.57)$ \\
\hline \multicolumn{5}{|l|}{ Age } \\
\hline$\leq 29$ & 1 & - & 1 & - \\
\hline $30-39$ & 1.28 & $(1.03-1.59)$ & 1.07 & $(0.87-1.32)$ \\
\hline $40-49$ & 1.26 & $(1.01-1.57)$ & 1.13 & $(0.93-1.37)$ \\
\hline $50-59$ & 1.57 & $(1.26-1.95)$ & 1.23 & $(1.01-1.49)$ \\
\hline $60 \leq$ & 1.68 & $(1.35-2.11)$ & 1.36 & $(1.09-1.70)$ \\
\hline \multicolumn{5}{|l|}{ Education } \\
\hline Middle school degree & 1 & - & 1 & - \\
\hline High School degree & 1.16 & $(1.00-1.35)$ & 1.02 & $(0.89-1.18)$ \\
\hline University degree or higher & 1.31 & $(1.10-1.57)$ & 0.99 & $(0.83-1.18)$ \\
\hline \multicolumn{5}{|l|}{ Income $^{a}$} \\
\hline Q1 & 0.98 & $(0.81-1.18)$ & 0.71 & $(0.60-0.85)$ \\
\hline Q2 & 1.01 & $(0.88-1.17)$ & 1.01 & $(0.87-1.17)$ \\
\hline$\hat{\mathrm{Q} 3}$ & 1.11 & $(0.98-1.26)$ & 1.03 & $(0.88-1.21)$ \\
\hline$\widehat{\mathrm{Q}} 4$ & 1 & - & 1 & - \\
\hline \multicolumn{5}{|l|}{ Depression } \\
\hline Yes & 2.44 & $(1.94-3.06)$ & 2.49 & $(2.06-3.00)$ \\
\hline No & 1 & - & 1 & - \\
\hline \multicolumn{5}{|l|}{ Fatigue } \\
\hline Yes & 1.06 & $(0.95-1.19)$ & 0.94 & $(0.86-1.04)$ \\
\hline No & 1 & - & 1 & - \\
\hline \multicolumn{5}{|l|}{ Hearing Problems } \\
\hline Yes & 1.49 & $(1.12-1.99)$ & 2.03 & $(1.52-2.72)$ \\
\hline No & 1 & - & 1 & - \\
\hline \multicolumn{5}{|l|}{ Headache/Eye strain } \\
\hline Yes & 1.28 & $(1.12-1.45)$ & 1.22 & $(1.09-1.37)$ \\
\hline No & 1 & - & 1 & - \\
\hline \multicolumn{5}{|l|}{ Subjective Health Condition } \\
\hline Good & 1 & - & 1 & - \\
\hline Normal & 1.43 & $(1.29-1.59)$ & 1.39 & $(1.27-1.53)$ \\
\hline Bad & 2.09 & $(1.71-2.57)$ & 2.22 & $(1.90-2.59)$ \\
\hline \multicolumn{5}{|l|}{ Physical Activity in Leisure Time } \\
\hline Everyday & 1 & - & 1 & - \\
\hline Several times per week & 1.03 & $(0.76-1.40)$ & 1.04 & $(0.76-1.42)$ \\
\hline Several times per month & 0.74 & $(0.55-1.00)$ & 0.72 & $(0.53-0.97)$ \\
\hline Rarely & 0.67 & $(0.50-0.91)$ & 0.55 & $(0.41-0.75)$ \\
\hline Never & 0.82 & $(0.61-1.10)$ & 0.69 & $(0.51-0.93)$ \\
\hline
\end{tabular}


Table 2. Cont.

\begin{tabular}{|c|c|c|c|c|}
\hline \multirow{3}{*}{ Variables } & \multicolumn{4}{|c|}{ Insomnia } \\
\hline & \multicolumn{2}{|c|}{ Male } & \multicolumn{2}{|c|}{ Female } \\
\hline & Adjusted OR & $95 \% \mathrm{CI}$ & Adjusted OR & $95 \% \mathrm{CI}$ \\
\hline \multicolumn{5}{|l|}{ Use of PPE ${ }^{b}$} \\
\hline Yes & 1 & - & 1 & - \\
\hline No & 1.39 & $(1.10-1.72)$ & 1.48 & $(1.19-1.83)$ \\
\hline Not applicable & 1.86 & $(1.65-2.08)$ & 1.33 & (1.19-1.48) \\
\hline \multicolumn{5}{|l|}{ Job Satisfaction } \\
\hline Yes & 1 & - & 1 & - \\
\hline No & 1.53 & $(1.38-1.69)$ & 1.73 & $(1.59-1.90)$ \\
\hline \multicolumn{5}{|l|}{ Work and life balance } \\
\hline Yes & 1 & - & 1 & - \\
\hline No & 1.16 & $(1.05-1.28)$ & 1.15 & $(1.04-1.26)$ \\
\hline \multicolumn{5}{|l|}{ Work duration } \\
\hline$\leq 5$ years & 1 & - & 1 & - \\
\hline $5-10$ years & 0.96 & $(0.85-1.08)$ & 1.06 & $(0.95-1.17)$ \\
\hline$\geq 11$ years & 0.86 & $(0.75-0.97)$ & 1.03 & $(0.92-1.16)$ \\
\hline \multicolumn{5}{|l|}{ Working hours/week } \\
\hline$\leq 40 \mathrm{~h}$ & 1 & - & 1 & - \\
\hline $41-50 \mathrm{~h}$ & 0.83 & $(0.72-0.96)$ & 0.9 & $(0.81-1.01)$ \\
\hline $51-60 \mathrm{~h}$ & 0.78 & $(0.67-0.91)$ & 0.68 & $(0.59-0.77)$ \\
\hline$\geq 61 \mathrm{~h}$ & 0.75 & $(0.63-0.90)$ & 0.64 & $(0.54-0.76)$ \\
\hline \multicolumn{5}{|l|}{ Job Collar ${ }^{c}$} \\
\hline White & 1 & - & 1 & - \\
\hline Blue & 1.01 & $(0.87-1.19)$ & 1.1 & $(0.93-1.29)$ \\
\hline Pink & 1.11 & $(0.95-1.30)$ & 1.12 & (0.98-1.29) \\
\hline \multicolumn{5}{|l|}{ Shift Work } \\
\hline Yes & 1.22 & $(1.04-1.42)$ & 1.19 & $(1.02-1.40)$ \\
\hline No & 1 & - & 1 & - \\
\hline \multicolumn{5}{|l|}{ Flexible break time } \\
\hline Yes & 1 & - & 1 & - \\
\hline No & 0.87 & $(0.78-0.98)$ & 0.73 & $(0.66-0.81)$ \\
\hline \multicolumn{5}{|l|}{ Size of Business } \\
\hline 1-9 people & 1.1 & $(0.84-1.45)$ & 0.89 & $(0.65-1.22)$ \\
\hline 10-249 people & 0.99 & $(0.75-1.31)$ & 0.89 & (0.64-1.23) \\
\hline$\geq 250$ people & 1 & - & 1 & - \\
\hline
\end{tabular}

In Table 3, additional logistic regression analyses were carried out to further investigate the association of insomnia with various occupational variables in workers who were exposed to noise and/or vibration. In men, those who were exposed to vibration and did not wear personal protective equipment when needed (OR 2.67; (95\% CI, 1.07-6.67)) as well as those working more than 11 years at their current job (OR 2.14; (95\% CI, 1.56-2.94)) had a high risk of insomnia. In addition, working in a business with 10 or fewer employees (OR 2.11; (95\% CI, 1.82-2.44)) whilst being exposed to simultaneous noise and vibration exposure had a strong correlation with insomnia as well. In women, those who were exposed to both noise and vibration and did not wear personal protective equipment (PPE) when required (OR 2.07; (95\% CI, 1.16-3.70)) and those working at a business with 10 or fewer employees (OR 3.56; (95\% CI, 3.10-4.10)) had an increased risk of insomnia. 
Table 3. The results of subgroup analysis stratified by occupational-related characteristics.

\begin{tabular}{|c|c|c|c|c|c|c|c|c|}
\hline \multirow{4}{*}{ Variables } & \multicolumn{8}{|c|}{ Insomnia } \\
\hline & \multicolumn{8}{|c|}{ Noise and Vibration Exposure } \\
\hline & \multirow{2}{*}{$\begin{array}{c}\text { No } \\
\begin{array}{c}\text { Adjusted } \\
\text { OR }\end{array}\end{array}$} & \multicolumn{2}{|c|}{ Noise Exposure } & \multicolumn{2}{|c|}{ Vibration Exposure } & \multicolumn{2}{|c|}{$\begin{array}{c}\text { Noise plus Vibration } \\
\text { Exposure }\end{array}$} & \multirow{2}{*}{$\begin{array}{l}p \text {-Value for } \\
\text { Trend }\end{array}$} \\
\hline & & $\begin{array}{l}\text { Adjusted } \\
\text { OR }\end{array}$ & $95 \% \mathrm{CI}$ & $\begin{array}{l}\text { Adjusted } \\
\text { OR }\end{array}$ & $95 \% \mathrm{CI}$ & $\begin{array}{l}\text { Adjusted } \\
\text { OR }\end{array}$ & $95 \% \mathrm{CI}$ & \\
\hline \multicolumn{9}{|l|}{ Male } \\
\hline \multicolumn{9}{|l|}{ Use of PPE ${ }^{a}$} \\
\hline Yes & 1 & 1.1 & $(0.85-1.44)$ & 1.24 & $(0.83-1.86)$ & 1.32 & $(1.06-1.66)$ & 0.09 \\
\hline No & 1 & 1.2 & $(0.70-2.07)$ & 2.67 & $(1.07-6.67)$ & 1.14 & $(0.68-1.92)$ & 0.075 \\
\hline Not applicable & 1 & 0.95 & $(0.76-1.18)$ & 1.97 & $(1.61-2.41)$ & 2.5 & $(2.13-2.95)$ & $<0.0001$ \\
\hline \multicolumn{9}{|l|}{ Job Satisfaction } \\
\hline Yes & 1 & 0.99 & $(0.81-1.20)$ & 2.07 & $(1.66-2.58)$ & 1.59 & $(1.34-1.88)$ & $<0.0001$ \\
\hline No & 1 & 1.26 & $(0.96-1.64)$ & 1.55 & $(1.15-2.09)$ & 2.13 & $(1.75-2.59)$ & $<0.0001$ \\
\hline \multicolumn{9}{|l|}{ Work and life balance } \\
\hline Yes & 1 & 1.09 & $(0.90-1.33)$ & 1.98 & $(1.57-2.50)$ & 1.8 & $(1.53-2.12)$ & $<0.0001$ \\
\hline No & 1 & 1.1 & $(0.84-1.42)$ & 1.64 & $(1.25-2.15)$ & 1.84 & $(1.50-2.26)$ & $<0.0001$ \\
\hline \multicolumn{9}{|l|}{ Work duration } \\
\hline$\leq 5$ years & 1 & 1.13 & $(0.82-1.56)$ & 1.87 & $(1.40-2.49)$ & 2.08 & $(1.66-2.61)$ & $<0.0001$ \\
\hline $5-10$ years & 1 & 1.21 & $(0.90-1.63)$ & 1.62 & $(1.17-2.24)$ & 2.16 & $(1.71-2.74)$ & $<0.0001$ \\
\hline$\geq 11$ years & 1 & 0.99 & $(0.79-1.25)$ & 2.14 & $(1.56-2.94)$ & 1.43 & $(1.16-1.75)$ & $<0.0001$ \\
\hline \multicolumn{9}{|l|}{ Working hours/week } \\
\hline$\leq 40 \mathrm{~h}$ & 1 & 1.45 & $(1.00-2.12)$ & 2.87 & $(1.86-4.41)$ & 3.46 & $(2.59-4.62)$ & $<0.0001$ \\
\hline $41-50 \mathrm{~h}$ & 1 & 1.04 & $(0.80-1.35)$ & 1.62 & $(1.20-2.18)$ & 1.6 & $(1.30-1.97)$ & 0.0001 \\
\hline $51-60 \mathrm{~h}$ & 1 & 1 & $(0.74-1.34)$ & 1.7 & $(1.22-2.37)$ & 1.41 & $(1.09-1.82)$ & 0.0014 \\
\hline$\geq 61 \mathrm{~h}$ & 1 & 1.01 & $(0.68-1.51)$ & 1.94 & $(1.25-3.00)$ & 1.57 & $(1.11-2.22)$ & 0.0019 \\
\hline \multicolumn{9}{|l|}{ Job Collar ${ }^{b}$} \\
\hline White & 1 & 1.22 & $(0.72-2.07)$ & 2.04 & $(1.33-3.12)$ & 1.72 & $(1.17-2.51)$ & 0.0002 \\
\hline Blue & 1 & 1.07 & $(0.89-1.29)$ & 1.93 & $(1.48-2.51)$ & 1.76 & $(1.51-2.05)$ & $<0.0001$ \\
\hline Pink & 1 & 1.05 & $(0.72-1.53)$ & 1.79 & $(1.33-2.40)$ & 2.58 & $(1.92-3.48)$ & $<0.0001$ \\
\hline \multicolumn{9}{|l|}{ Shift Work } \\
\hline Yes & 1 & 1.54 & $(0.90-2.62)$ & 1.66 & $(1.04-2.64)$ & 1.44 & $(0.94-2.19)$ & 0.0141 \\
\hline No & 1 & 1.07 & $(0.90-1.26)$ & 1.86 & $(1.54-2.26)$ & 1.86 & $(1.62-2.12)$ & $<0.0001$ \\
\hline \multicolumn{9}{|l|}{ Flexible break time } \\
\hline Yes & 1 & 1.1 & $(0.92-1.31)$ & 2.07 & $(1.70-2.53)$ & 2.03 & $(1.76-2.34)$ & $<0.0001$ \\
\hline No & 1 & 1.09 & $(0.77-1.55)$ & 1.15 & $(0.78-1.71)$ & 1.21 & $(0.90-1.61)$ & 0.3698 \\
\hline \multicolumn{9}{|l|}{ Size of Business } \\
\hline 1-9 people & 1 & 1.15 & $(0.96-1.37)$ & 1.89 & $(1.54-2.34)$ & 2.11 & $(1.82-2.44)$ & $<0.0001$ \\
\hline 10-249 people & 1 & 1.03 & $(0.71-1.50)$ & 1.76 & $(1.25-2.49)$ & 1.26 & $(0.96-1.66)$ & 0.0019 \\
\hline$\geq 250$ people & 1 & 0.35 & $(0.09-1.33)$ & 1.38 & $(0.37-5.14)$ & 1.31 & $(0.59-2.92)$ & 0.6837 \\
\hline \multicolumn{9}{|l|}{ Female } \\
\hline \multicolumn{9}{|l|}{ Use of PPE ${ }^{a}$} \\
\hline Yes & 1 & 0.98 & $(0.74-1.31)$ & 1.33 & $(0.92-1.93)$ & 1.54 & $(1.17-2.02)$ & 0.0469 \\
\hline No & 1 & 0.99 & $(0.51-1.92)$ & 1.37 & $(0.54-3.48)$ & 2.07 & $(1.16-3.70)$ & 0.0534 \\
\hline Not applicable & 1 & 1.1 & $(0.89-1.36)$ & 1.69 & $(1.40-2.03)$ & 4.45 & $(3.80-5.21)$ & $<0.0001$ \\
\hline \multicolumn{9}{|l|}{ Job Satisfaction } \\
\hline Yes & 1 & 1.09 & $(0.89-1.33)$ & 1.71 & $(1.39-2.11)$ & 3.01 & $(2.54-3.55)$ & $<0.0001$ \\
\hline No & 1 & 1.02 & $(0.76-1.36)$ & 1.39 & $(1.07-1.81)$ & 3.15 & $(2.57-3.87)$ & $<0.0001$ \\
\hline
\end{tabular}


Table 3. Cont.

\begin{tabular}{|c|c|c|c|c|c|c|c|c|}
\hline \multirow{4}{*}{ Variables } & \multicolumn{8}{|c|}{ Insomnia } \\
\hline & \multicolumn{8}{|c|}{ Noise and Vibration Exposure } \\
\hline & \multirow{2}{*}{$\begin{array}{c}\text { No } \\
\begin{array}{c}\text { Adjusted } \\
\text { OR }\end{array}\end{array}$} & \multicolumn{2}{|c|}{ Noise Exposure } & \multicolumn{2}{|c|}{ Vibration Exposure } & \multicolumn{2}{|c|}{$\begin{array}{c}\text { Noise plus Vibration } \\
\text { Exposure }\end{array}$} & \multirow{2}{*}{$\begin{array}{l}p \text {-Value for } \\
\text { Trend }\end{array}$} \\
\hline & & $\begin{array}{l}\text { Adjusted } \\
\text { OR }\end{array}$ & $95 \%$ CI & $\begin{array}{l}\text { Adjusted } \\
\text { OR }\end{array}$ & $95 \%$ CI & $\begin{array}{l}\text { Adjusted } \\
\text { OR }\end{array}$ & $95 \%$ CI & \\
\hline \multicolumn{9}{|l|}{ Work and life balance } \\
\hline Yes & 1 & 1.09 & $(0.89-1.33)$ & 1.58 & $(1.28-1.96)$ & 3.16 & $(2.69-3.72)$ & $<0.0001$ \\
\hline No & 1 & 1.07 & $(0.81-1.42)$ & 1.62 & $(1.25-2.10)$ & 3.05 & $(2.46-3.77)$ & $<0.0001$ \\
\hline \multicolumn{9}{|l|}{ Work duration } \\
\hline$\leq 5$ years & 1 & 1.02 & $(0.75-1.40)$ & 1.59 & $(1.24-2.04)$ & 3.79 & $(3.07-4.67)$ & $<0.0001$ \\
\hline $5-10$ years & 1 & 0.94 & $(0.67-1.30)$ & 1.92 & $(1.46-2.54)$ & 3.28 & $(2.56-4.20)$ & $<0.0001$ \\
\hline$\geq 11$ years & 1 & 1.17 & $(0.92-1.49)$ & 1.24 & $(0.87-1.78)$ & 2.54 & $(2.03-3.17)$ & $<0.0001$ \\
\hline \multicolumn{9}{|l|}{ Working hours/week } \\
\hline$\leq 40 \mathrm{~h}$ & 1 & 1.17 & $(0.86-1.59)$ & 1.39 & $(1.03-1.87)$ & 5.77 & $(4.58-7.27)$ & $<0.0001$ \\
\hline $41-50 \mathrm{~h}$ & 1 & 1.25 & $(0.93-1.67)$ & 1.83 & $(1.37-2.44)$ & 2.37 & $(1.87-2.99)$ & $<0.0001$ \\
\hline $51-60 \mathrm{~h}$ & 1 & 0.85 & $(0.61-1.19)$ & 2.01 & $(1.46-2.76)$ & 2.11 & $(1.59-2.78)$ & $<0.0001$ \\
\hline$\geq 61 \mathrm{~h}$ & 1 & 0.97 & $(0.60-1.57)$ & 1.01 & $(0.60-1.71)$ & 3.16 & $(1.99-5.03)$ & 0.1118 \\
\hline \multicolumn{9}{|l|}{ Job Collar ${ }^{b}$} \\
\hline White & 1 & 1.16 & $(0.64-2.09)$ & 2.72 & $(1.85-4.02)$ & 2.92 & $(1.89-4.50)$ & $<0.0001$ \\
\hline Blue & 1 & 1.09 & $(0.87-1.36)$ & 1.28 & $(0.92-1.80)$ & 2.93 & $(2.43-3.54)$ & $<0.0001$ \\
\hline Pink & 1 & 1.07 & $(0.81-1.41)$ & 1.56 & $(1.26-1.94)$ & 3.76 & $(3.07-4.60)$ & $<0.0001$ \\
\hline \multicolumn{9}{|l|}{ Shift Work } \\
\hline Yes & 1 & 0.94 & $(0.42-2.15)$ & 1.44 & $(0.85-2.44)$ & 1.48 & $(0.83-2.66)$ & 0.2207 \\
\hline No & 1 & 1.09 & $(0.92-1.29)$ & 1.6 & $(1.35-1.90)$ & 3.25 & $(2.85-3.71)$ & $<0.0001$ \\
\hline Flexible break time & 1 & & & & & & & \\
\hline Yes & 1 & 1.09 & $(0.91-1.30)$ & 1.59 & $(1.32-1.92)$ & 3.67 & $(3.18-4.24)$ & $<0.0001$ \\
\hline No & 1 & 0.98 & $(0.67-1.44)$ & 1.53 & $(1.10-2.12)$ & 1.64 & $(1.20-2.26)$ & 0.0021 \\
\hline \multicolumn{9}{|l|}{ Size of Business } \\
\hline 1-9 people & 1 & 1.06 & $(0.89-1.27)$ & 1.62 & $(1.35-1.95)$ & 3.56 & $(3.10-4.10)$ & $<0.0001$ \\
\hline 10-249 people & 1 & 1.34 & $(0.84-2.13)$ & 1.52 & $(1.04-2.22)$ & 1.82 & $(1.29-2.59)$ & 0.0019 \\
\hline$\geq 250$ people & 1 & 0.23 & $(0.03-1.68)$ & 1.16 & $(0.28-4.89)$ & 0.42 & $(0.10-1.69)$ & 0.5928 \\
\hline
\end{tabular}

Adjusted for other covariates; ${ }^{a}$ Personal Protective Equipment; ${ }^{\mathrm{b}}$ Job collar types classified according the Korean Standard Occupational Classification.

Table 4 presents the results of subgroup analysis indicating the degree of exposure to noise and/or vibration exposure and the association with insomnia. Both male and female workers showed a dose-response relationship for noise only and vibration only exposure to a certain extent as shown in the following: "Degree of noise exposure" in men gradually increased from 1 to 3 points (OR 1.22 (95\% CI, 1.06-1.40); OR 1.67 (95\% CI, 1.41-1.98); OR 1.77 (95\% CI, 1.43-1.98)) and then decreased from 4 to 5 points (OR 1.57 (95\% CI, 1.27-1.94); OR 0.96 (95\% CI, 0.71-1.31)). In women, it gradually increased from 1 to 4 points (OR 1.57 (95\% CI, 1.36-1.81); OR 2.13 (95\% CI, 1.75-2.58); OR 2.86 (95\% CI, 2.24-3.65); OR 2.97 (95\% CI, 2.29-3.83)) and then decreased at 5 points (OR 1.31 (95\% CI, 1.27-1.94)). For "degree of vibration exposure", a similar trend can be seen in men from 1 to 4 points (OR 1.54 (95\% CI, 1.35-1.76); OR 2.10 (95\% CI, 1.75-2.53); OR 2.17 (95\% CI, 1.74-2.71); OR 2.25 (95\% CI, 1.75-2.89)) and decreased at 5 points (OR 1.83 (95\% CI, 1.24-2.69). In women, it increased from 1 to 4 points (OR $1.80(95 \% \mathrm{CI}$, 1.57-2.06); OR 2.94 (95\% CI, 2.40-3.59); OR 3.74 (95\% CI, 2.86-4.88); OR 4.68 (95\% CI, 3.49-6.28)) and then decreased at 5 points (OR 1.82 (95\% CI, 1.04-3.17)). For "degree of noise plus vibration exposure", a dose-response relationship could be seen in both males and females. The OR of insomnia increased with increasing degree of exposure by 1 to 3 points, 4 to 5 points, and 6 to 8 points in men with OR 1.44 (95\% CI, 1.29-1.62); OR 1.88 (95\% CI, 1.58-2.24); and OR 2.05 (95\% CI, 1.69-2.48), respectively, and 
then decreased at 9 to 10 points (OR 1.37 (95\% CI, 0.91-2.04)), and in women, it increased with OR 1.57 (95\% CI, 1.41-1.74); OR 2.24 (95\% CI, 1.83-2.73); and OR 5.25 (95\% CI, 4.12-6.67), respectively, and then decreased at 9 to 10 points (OR 2.69 (95\% CI, 1.40-5.16)).

Table 4. The results of degree of noise and/or vibration exposure with insomnia.

\begin{tabular}{|c|c|c|c|c|c|c|}
\hline \multirow{3}{*}{ Variables } & \multicolumn{6}{|c|}{ Insomnia } \\
\hline & \multicolumn{2}{|c|}{ Male } & \multirow{2}{*}{$\begin{array}{l}p \text {-Value for } \\
\text { Trend }\end{array}$} & \multicolumn{2}{|c|}{ Female } & \multirow{2}{*}{$\begin{array}{c}p \text {-Value for } \\
\text { Trend }\end{array}$} \\
\hline & $\begin{array}{c}\text { Adjusted } \\
\text { OR }\end{array}$ & $95 \% \mathrm{CI}$ & & $\begin{array}{c}\text { Adjusted } \\
\text { OR }\end{array}$ & $95 \%$ CI & \\
\hline Degree of Noise Exposure $^{\text {a }}$ & & & $<0.0001$ & & & $<0.0001$ \\
\hline 0 & 1 & - & & 1 & - & \\
\hline 1 & 1.22 & $(1.06-1.40)$ & & 1.57 & $(1.36-1.81)$ & \\
\hline 2 & 1.67 & $(1.41-1.98)$ & & 2.13 & $(1.75-2.58)$ & \\
\hline 3 & 1.77 & $(1.43-2.21)$ & & 2.86 & $(2.24-3.65)$ & \\
\hline 4 & 1.57 & $(1.27-1.94)$ & & 2.97 & $(2.29-3.83)$ & \\
\hline 5 & 0.96 & $(0.71-1.31)$ & & 1.31 & $(0.84-2.04)$ & \\
\hline Degree of Vibration Exposure ${ }^{a}$ & & & $<0.0001$ & & & $<0.0001$ \\
\hline 0 & 1 & - & & 1 & - & \\
\hline 1 & 1.54 & $(1.35-1.76)$ & & 1.8 & $(1.57-2.06)$ & \\
\hline 2 & 2.1 & $(1.75-2.53)$ & & 2.94 & $(2.40-3.59)$ & \\
\hline 3 & 2.17 & $(1.74-2.71)$ & & 3.74 & $(2.86-4.88)$ & \\
\hline 4 & 2.25 & $(1.75-2.89)$ & & 4.68 & $(3.49-6.28)$ & \\
\hline 5 & 1.83 & $(1.24-2.69)$ & & 1.82 & $(1.04-3.17)$ & \\
\hline Degree of Vibration + Noise Expc & & & $<0.0001$ & & & $<0.0001$ \\
\hline 0 & 1 & - & & 1 & - & \\
\hline 1 to 3 & 1.44 & $(1.29-1.62)$ & & 1.57 & $(1.41-1.74)$ & \\
\hline 4 to 5 & 1.88 & $(1.58-2.24)$ & & 2.24 & $(1.83-2.73)$ & \\
\hline 6 to 8 & 2.05 & $(1.69-2.48)$ & & 5.25 & $(4.12-6.67)$ & \\
\hline 9 to 10 & 1.37 & $(0.91-2.04)$ & & 2.69 & $(1.40-5.16)$ & \\
\hline
\end{tabular}

${ }^{a}$ Both degree of noise exposure only and vibration exposure only were assigned to a grade point scale with 0 points being; no exposure at all and 5 points being the maximum indicating exposure all the time; ${ }^{b}$ The total combined points of noise exposure and vibration exposure with 0 points being the minimum and 10 points being the maximum total degree of exposure.

Table 5 shows the results of the synergistic effects of simultaneous noise and vibration exposure on insomnia risk. The relative excess risk due to interaction (RERI), attributable proportion (AP), and synergy index (SI) for the combination were $-0.10,-0.06$, and 0.75 in men and $1.49,0.47$, and 1.60 in women, respectively. In males, none of the values were statistically significant. Therefore, no synergistic effect was seen. However, in females, RERI and AP values were greater than zero and SI exceeded one, and were statistically significant, signifying synergistic interaction. 
Table 5. Additive interaction of noise and vibration exposure on insomnia.

\begin{tabular}{|c|c|c|}
\hline \multirow{3}{*}{ Additive Interaction } & \multicolumn{2}{|c|}{ Insomnia } \\
\hline & \multicolumn{2}{|c|}{ Noise plus Vibration Exposure } \\
\hline & Adjusted OR & $95 \% \mathrm{CI}$ \\
\hline \multicolumn{3}{|l|}{ Male } \\
\hline RERI $^{a}$ & -0.1 & $(-0.49-0.28)$ \\
\hline$A P^{b}$ & -0.06 & $(-0.44-0.33)$ \\
\hline Synergy Index & 0.75 & $(0.37-1.14)$ \\
\hline \multicolumn{3}{|l|}{ Female } \\
\hline RERI $^{a}$ & 1.49 & $(1.02-1.96)$ \\
\hline $\mathbf{A P} \mathbf{b}^{\mathbf{b}}$ & 0.47 & $(0.00-0.94)$ \\
\hline Synergy Index & 1.6 & $(1.13-2.07)$ \\
\hline
\end{tabular}

\section{Discussion}

This study used the 5th Korean Working Conditions Survey (KWCS) to analyze the association between occupational noise and vibration exposure and insomnia. Our findings suggest that more than noise exposure, exposure to vibration had a prominent effect on insomnia. In addition, the prevalence of insomnia was higher in females than in males, which is consistent with previous findings that women are more vulnerable to mental health problems than men $[25,26]$. However, with the exception of simultaneous noise and vibration exposure, the OR of insomnia was higher in males than females. This could be explained by prior studies reporting that men are more likely to be exposed to hazardous occupational conditions than women, thereby making them more susceptible to their detrimental effects $[27,28]$. Previous studies indicated that long-term exposure to noise or vibration can endlessly stimulate the autonomic nervous system [29], causing sustained activation of the central autonomic system and induction of sympathetic nervous activity [30]. Insomnia may arise from stimulation of the peripheral nervous system [31,32]. Another study showed that workers exposed to vibration from operating heavy-duty machinery or working inside buildings resulted in an imbalance in the sympathetic nervous system [33]. It was previously revealed that chronic vibration exposure had a significant effect on poor sleep, independent of noise level exposure [33,34].

Prior laboratory research regarding noise exposure effects on sleep has shown ambiguous results. It seems that noise exposure effects are complex, and the absence of a clear dose-effect relationship is due to several factors including noise severity and the individual's sensitivity. Another study suggested that the effect of noise exposure on insomnia may eventually be habituated, thereby offering another explanation as to why the effect was not as prominently shown in this study [35].

The synergistic effect of noise and vibration has been proven in prior studies on health outcomes such as hearing loss [36,37], headache/eyestrain [7], and cognitive performance [38]. The rationale behind investigating the effect of both noise and vibration with insomnia is that, for instance, when handling large equipment or driving large vehicles, workers are often exposed to noise and vibration simultaneously in their work environments. A prior study carried out in Korea showed that combined noise and vibration exposure had a greater effect on the increased total of nervous system-related disorders, which included headache/eyestrain, fatigue, and sleep disturbance/insomnia [9]. Therefore, there is a need to investigate the combined effect of noise and vibration. Through the results of interaction analysis, the synergistic effect of both combined noise and vibration exposure on insomnia was seen in females, but not in males, supporting prior research [39]. A hypothesis pertaining to the reason why the synergistic effects were not seen in men could be attributed to the healthy worker effect, whereby male workers who were more likely to be exposed to severe hazardous occupational factors 
could not tolerate working conditions where simultaneous noise and vibration exposure occurred and either quit their jobs or retired.

Regarding the subgroup analysis of occupational variables and noise and vibration exposure in individuals with insomnia, men and women who did not wear PPE when required and were exposed to vibration exposure only or both noise and vibration exposure, therefore eliminating a form of protection against the two exposures, had a significantly higher risk of insomnia supporting the findings of a prior study [9]. The implications of not wearing specialized PPE such as earplugs for noise exposure or anti-vibration gloves for vibration exposure can be severe; therefore, the World Health Organization (WHO) released guidelines regarding PPE use to lessen these harmful effects [40]. Additionally, men and women who worked in workplaces with 10 or fewer employees had a higher risk of insomnia compared to those who worked larger businesses. This could be linked to the previous point in the sense that smaller workplaces are not as fully equipped and prepared for safety protocols against exposure to these occupational hazards including the provision of PPE.

In addition, men who were exposed to vibration and worked more than 11 years had a higher risk of insomnia. This was consistent with a prior study that speculated that chronic exposure to vibration may result in a constant state of hyperarousal of the autonomic nervous system, leading to psychological disorders including disturbed sleep [41].

A dose-response relationship could be inferred from the variable of interest subgroup analysis presenting the relationship of the extent of noise and vibration exposure both separately and combined with insomnia. In noise exposure and vibration exposure, individually and combined, with increasing time spent being exposed to these factors, the risk of insomnia gradually increased, signifying a dose-response relationship. However, when the exposure was 4 or 5 points or, in the case of simultaneous exposure, 9 to 10 points, which indicated exposure to either noise or vibration almost all or all the time, the OR of insomnia suddenly decreased. This could again be explained due to the phenomenon of the healthy worker effect, where those who suffered severe health consequences or could not tolerate constant exposure to noise or vibration quit, retired, or changed occupations.

The following limitations were recognized in our study. First, as the obtained data were analyzed cross-sectionally, a causal relationship could not be verified. Second, this study involved self-reported questionnaires; therefore, we could not rule out the possibility of response and recall bias. Third, information provided in this survey was lacking key variables such as smoking and drinking habits. Fourth, different types of vibration exist, such as whole-body vibration and hand-arm vibration [42], but they were not specified in the survey. Fifth, we could not investigate people who experienced early-morning awakening with the inability to return to sleep, as there was limited information on sleep variables. On the other hand, the most commonly reported symptom of insomnia was reported to be difficulty maintaining sleep followed by difficulty in initiating sleep [43,44]. Finally, there was a lack of objective assessment in regard to both exposure and outcome evaluation. For example, we could not quantify the amount of noise and vibration exposure in the workplace and could not use more reliable measures of assessment of sleep problems, e.g., polysomnography.

On the other hand, our study's main strong point is that, to the extent of our knowledge, this is the first study in South Korea and one of the few studies worldwide to focus on the simultaneous exposure of noise and vibration effects on insomnia. Other studies have previously investigated the effect of noise and vibration exposure on a variety of mental health problems [9,45]. However, those exposures were investigated individually, not simultaneously. In addition, another strength that sets apart our study is that we used interaction analysis to evaluate the effects of synergistic exposure to noise and vibration on insomnia risk. Although previous studies have previously investigated the synergistic effect of noise and vibration, those studies mainly investigated their effects on hearing loss $[46,47]$. Another strength lies in the fact that we investigated the effects of noise and vibration exposure on insomnia in a nationally inclusive sample of Korean workers, and it was stratified by sex. 


\section{Methods}

Our study used data obtained from the 2017 Korean Working Conditions Survey led by the Korea Occupational Safety and Health Agency (KOSHA). Since the first KWCS survey in 2006, statistical data have been periodically obtained on Korean workers' health-related characteristics as well as occupational risk factors. A multistage random-sampling approach based on the Population and Housing Census was used in the KWCS and could be considered to be representative of the overall population of Korean workers. The survey data were collected through direct interviews through house visits, the target population being workers aged $\geq 15$ years old. In the event where there was more than one eligible employee, the interviewers carried out interviews with those whose birth date was closest to the research date. Information was obtained about each employee's general characteristics, occupational characteristics, and state of health. All participants provided written informed consent and were guaranteed anonymity. In the 5th edition of the Korean Working Conditions Survey, a total of 50,027 participants were included. After excluding those with missing data or those who failed or refused to respond, a final population of 30,837 people was selected for this study.

In this study, the dependent variable in question was insomnia. The classification used to examine the presence of insomnia was based on the fifth edition of the Diagnostic and Statistical Manual of Mental Disorders (DSM-5) [44]. The diagnostic criteria for insomnia included those who experience one or more of the following symptoms at least three nights per week for at least three months: (1) difficulty initiating sleep, (2) difficulty maintaining sleep, and (3) early-morning awakening with the inability to return to sleep. The KWCS questionnaire included a question that asked, "Over the past 12 months, did you suffer from sleep-related problems?", and those who answered "daily", "several times a week", or "several times a month" in response to at least one of the aforementioned symptoms were determined to have insomnia.

The variable of interest in this study was noise and vibration exposure. Noise and vibration exposure were assessed by the following two questions: "In your workplace, are you exposed to noise so loud that you have to raise your voice to keep a conversation during work?" and "How much are you exposed to hand-transmitted vibration or vibration generated by machinery?" Seven responses were possible depending on the time spent being exposed to the aforementioned ergonometric factors: never, almost never, one-quarter of the time, half of the time, three-quarters of the time, almost all the time, and all of the time. These were then clustered dichotomously, as follows: "never" and "almost never" was reclassified as "no exposure", and the rest of the responses were grouped into the exposed group for noise, vibration, and noise plus vibration exposure. In the subgroup analysis of our variable of interest, the degree of noise and vibration was each classified using a grade-point system. Zero points were the combined responses of "never" and "almost never", one point was "one-quarter of the time", two points was "half of the time", "three-quarters of the time" was three points, four points was "almost all the time", and the maximum of five points was given to "all of the time." Noise plus vibration exposure was the combined total of the noise and vibration exposure degree and was grouped into five categories "zero points", "one to three points", "four to five points", "six to eight points", and "9 to 10 points".

Various sociodemographic, health-related, and occupational characteristics were all added as potential confounding variables in this study. Sociodemographic characteristics included the following: gender, age ( $\leq 29,30-39,40-49,50-59, \geq 60)$, education level (elementary school degree or lower, middle school degree, high school degree, university degree or higher), and income level per month, which was divided into four quartiles $(<150,000,<250,000,<350,000, \geq 350,000)$. Health-related variables encompassed the following: depression, fatigue, presence of hearing problems, presence of headache/eyestrain symptoms, subjective health condition (good, normal, bad), and physical activity in leisure time (every day, several times per week, several times per month, rarely, never). Occupational-related variables included the following: use of PPE including earplugs, helmets, etc., job satisfaction (very satisfied, satisfied, a little unsatisfied, unsatisfied), work and life balance, work duration ( $\leq 5$ years, 5-10 years, $\geq 11$ years), and working hours per week ( $\leq 40 \mathrm{~h}, 41-50 \mathrm{~h}, 51-60 \mathrm{~h}$, 
$\geq 61 \mathrm{~h}$ ). Job types were based on the Korean Standard Occupational Classification (6th revision) classified according to three categories: white collar (administrators, professionals, engineers and semi-professionals, and office workers), pink collar (service workers and sales workers), and blue collar (skilled agricultural, forestry, and fishery industry workers; technically skilled worker operators and related skill workers; equipment or machinery operator and assembly workers; and simple laborers) [48]. Other occupational-related variables included shift work, flexible break time, and lastly, size of business (1-9 people, $10-249$ people, and $\geq 250$ people).

A chi-squared test was utilized to compare the covariates of the study participants. The association between noise and vibration exposure and insomnia in workers was analyzed via multiple logistic regression, and p-values less than 0.05 were statistically significant. In the subgroup analysis, the association between occupational-related variables and insomnia, as well as the trend for significance between the degree of noise and vibration exposure with insomnia, was carried out and confirmed through p-value for trend analysis. P-values for trend results less than 0.05 were considered statistically significant.

Additive interaction analysis to examine the interaction between noise and vibration exposure and insomnia was carried out. Three measures of additive interactions-RERI, AP, and SI-and their 95\% CI were calculated. If RERI and AP did not equal zero and SI exceeded one, then additive interaction was considered present. In addition, if RERI was greater than zero, the interaction was considered synergistic; if RERI was less than zero, an antagonistic interaction was implied. All statistical analyses were conducted using SAS 9.4 software (SAS Inc., Cary, NC, USA).

\section{Conclusions}

In conclusion, our findings suggested an association between workplace noise and vibration exposure and insomnia. From a public health viewpoint, it is important to tackle and address problems affecting the sleep quality of these workers as it negatively impacts workers' health and quality of life and performance in the workplace.

Author Contributions: Formal analysis, F.N., Y.K.K., and S.H.K.; supervision, E.-C.P. and S.-I.J.; conceptualization, Y.K.K.; visualization, Y.K.K. and F.N.; writing-original draft, F.N.; writing-review and editing, E.-C.P. and S.-I.J. All authors have read and agreed to the published version of the manuscript.

Funding: This study was supported by a faculty research grant of Yonsei University College of Medicine (6-2018-0174 and 6-2017-0157).

Acknowledgments: We would like to thank the Safety and Health Policy Research Department (Occupational Safety and Health Research Institute) for providing the raw data from the Korean Working Conditions Survey. The paper's contents are solely the responsibility of the authors and do not necessarily represent the official views of the OSHRI.

Conflicts of Interest: The authors declare no conflict of interest.

\section{References}

1. Pyykko, I.; Farkkila, M.; Inaba, R.; Starck, J.; Pekkarinen, J. Effect of hand-arm vibration on inner ear and cardiac functions in man. Nagoya J. Med. Sci. 1994, 57, 113-119. [PubMed]

2. Erdogan, E.; Yazgan, M.E. Landscaping in reducing traffic noise problem in cities: Ankara case. Afr. J. Agric. Res. 2009, 4, 1015-1022.

3. Takeuchi, T.; Futatsuka, M.; Imanishi, H.; Yamada, S. Pathological changes observed in the finger biopsy of patients with vibration-induced white finger. Scand. J. Work Environ. Health 1986, 12, 280-283. [CrossRef] [PubMed]

4. Lang, T.; Fouriaud, C.; Jacquinet-Salord, M.-C. Length of occupational noise exposure and blood pressure. Int. Arch. Occup. Environ. Health 1992, 63, 369-372. [CrossRef]

5. Ising, H.; Kruppa, B. Health effects caused by noise: Evidence in the literature from the past 25 years. Noise Health 2003, 6, 5-13.

6. Passchier-Vermeer, W.; Passchier, W.F. Noise exposure and public health. Environ. Health Perspect. 2000, 108 (Suppl. S1), 123-131. 
7. Kim, J.; Lee, W.; Won, J.-U.; Yoon, J.-H.; Seok, H.; Kim, Y.-K.; Lee, S.; Roh, J. The relationship between occupational noise and vibration exposure and headache/eyestrain, based on the fourth Korean Working Condition Survey (KWCS). PLOS ONE 2017, 12, e0177846. [CrossRef]

8. Jiao, K.; Li, Z.; Chen, M.; Wang, C.; Qi, S. Effect of different vibration frequencies on heart rate variability and driving fatigue in healthy drivers. Int. Arch. Occup. Environ. Health 2004, 77, 205-212. [CrossRef]

9. Lee, S.; Lee, W.; Roh, J.; Won, J.-U.; Yoon, J.-H. Symptoms of Nervous System Related Disorders Among Workers Exposed to Occupational Noise and Vibration in Korea. J. Occup. Environ. Med. 2017, 59, 191-197. [CrossRef]

10. Stansfeld, S.A.; Matheson, M.P. Noise pollution: Non-auditory effects on health. Br. Med Bull. 2003, 68, 243-257. [CrossRef]

11. Evandt, J.; Oftedal, B.; Hjertager Krog, N.; Nafstad, P.; Schwarze, P.E.; Marit Aasvang, G. A Population-Based Study on Nighttime Road Traffic Noise and Insomnia. Sleep 2016, 40. [CrossRef] [PubMed]

12. Muzet, A. Environmental noise, sleep and health. Sleep Med. Rev. 2007, 11, 135-142. [CrossRef] [PubMed]

13. Halperin, D. Environmental noise and sleep disturbances: A threat to health? Sleep Sci. 2014, 7, $209-212$. [CrossRef] [PubMed]

14. Ohayon, M.M. Epidemiology of insomnia: What we know and what we still need to learn. Sleep Med. Rev. 2002, 6, 97-111. [CrossRef]

15. Miedema, H.M.E.; Vos, H. Associations between Self-Reported Sleep Disturbance and Environmental Noise Based on Reanalyses of Pooled Data from 24 Studies. Behav. Sleep Med. 2007, 5, 1-20. [CrossRef]

16. Bonnet, M.H.; Arand, D.L. Clinical effects of sleep fragmentation versus sleep deprivation. Sleep Med. Rev. 2003, 7, 297-310. [CrossRef]

17. Martin, S.E.; Engleman, H.M.; Deary, I.J.; Douglas, N.J. The effect of sleep fragmentation on daytime function. Am. J. Respir. Crit. Care Med. 1996, 153, 1328-1332. [CrossRef]

18. Granö, N.; Vahtera, J.; Virtanen, M.; Keltikangas-Järvinen, L.; Kivimäki, M. Association of hostility with sleep duration and sleep disturbances in an employee population. Int. J. Behav. Med. 2008, 15, 73-80. [CrossRef]

19. Daley, M.; Morin, C.M.; LeBlanc, M.; Grégoire, J.P.; Savard, J.; Baillargeon, L. Insomnia and its relationship to health-care utilization, work absenteeism, productivity and accidents. Sleep Med. 2009, 10, 427-438. [CrossRef]

20. Léger, D.; Bayon, V.; Ohayon, M.M.; Philip, P.; Ement, P.; Metlaine, A.; Chennaoui, M.; Faraut, B. Insomnia and accidents: Cross-sectional study (EQUINOX) on sleep-related home, work and car accidents in 5293 subjects with insomnia from 10 countries. J. Sleep Res. 2014, 23, 143-152. [CrossRef]

21. Berglund, B.; Lindvall, T.; Schwela, D.H.; World Health Organization. Guidelines for Community Noise; World Health Organization: Geneva, Switzerland, 1999.

22. Seong, J.C.; Park, T.H.; Ko, J.H.; Chang, S.I.; Kim, M.; Holt, J.B.; Mehdi, M.R. Modeling of road traffic noise and estimated human exposure in Fulton County, Georgia, USA. Environ. Int. 2011, 37, 1336-1341. [CrossRef]

23. Lee, H.-S.; Choi, S.-B. Control and Response Characteristics of a Magneto-Rheological Fluid Damper for Passenger Vehicles. J. Intell. Mater. Syst. Struct. 2000, 11, 80-87. [CrossRef]

24. Griffin, M.J. Measurement, evaluation, and assessment of occupational exposures to hand-transmitted vibration. Occup. Environ. Med. 1997, 54, 73-89. [CrossRef] [PubMed]

25. La, Y.K.; Choi, Y.H.; Chu, M.K.; Nam, J.M.; Choi, Y.-C.; Kim, W.-J. Gender differences influence over insomnia in Korean population: A cross-sectional study. PLoS ONE 2020, 15, e227190. [CrossRef] [PubMed]

26. Kim, S.M.; Jung, J.-W.; Park, I.-W.; Ahn, C.M.; Kim, Y.-I.; Yoo, K.-H.; Chun, E.M.; Jung, J.Y.; Park, Y.S.; Park, J.H.; et al. Gender Differences in Relations of Smoking Status, Depression, and Suicidality in Korea: Findings from the Korea National Health and Nutrition Examination Survey 2008-2012. Psychiatry Investig. 2016, 13, 239-246. [CrossRef]

27. Lee, W.; Lee, J.-G.; Yoon, J.-H.; Lee, J.-H. Relationship between occupational dust exposure levels and mental health symptoms among Korean workers. PLoS ONE 2020, 15, e0228853. [CrossRef] [PubMed]

28. Park, S.J.; Jung, M.; Sung, J.H. Influence of Physical and Musculoskeletal Factors on Occupational Injuries and Accidents in Korean Workers Based on Gender and Company Size. Int. J. Environ. Res. Public Health 2019, 16, 345. [CrossRef]

29. Seidman, M.D.; Standring, R.T. Noise and quality of life. Int. J. Environ. Res. Public Health 2010, 7, 3730-3738. [CrossRef] 
30. Yoon, J.-H.; Won, J.-U.; Lee, W.; Jung, P.K.; Roh, J. Occupational noise annoyance linked to depressive symptoms and suicidal ideation: A result from nationwide survey of Korea. PLoS ONE 2014, 9, e105321. [CrossRef]

31. Hardoy, M.C.; Carta, M.G.; Marci, A.R.; Carbone, F.; Cadeddu, M.; Kovess, V.; Dell'Osso, L.; Carpiniello, B. Exposure to aircraft noise and risk of psychiatric disorders: The Elmas survey-Aircraft noise and psychiatric disorders. Soc. Psychiatry Psychiatr. Epidemiol. 2005, 40, 24-26. [CrossRef]

32. Murata, K.; Araki, S.; Maeda, K. Autonomic and peripheral nervous system dysfunction in workers exposed to hand-arm vibration: A study of R-R interval variability and distribution of nerve conduction velocities. Int. Arch. Occup. Environ. Health 1991, 63, 205-211. [CrossRef] [PubMed]

33. Harada, N. Autonomic nervous function of hand-arm vibration syndrome patients. Nagoya J. Med. Sci. 1994, 57,77-85. [PubMed]

34. Smith, M.G.; Croy, I.; Ögren, M.; Persson Waye, K. On the Influence of Freight Trains on Humans: A Laboratory Investigation of the Impact of Nocturnal Low Frequency Vibration and Noise on Sleep and Heart Rate. PLoS ONE 2013, 8, e55829. [CrossRef] [PubMed]

35. Griefahn, B. Sleep disturbances related to environmental noise. Noise Health 2002, 4, 57-60.

36. Zhu, S.-K.; Sakakibara, H.; Yamada, S.Y. Combined effects of hand-arm vibration and noise on temporary threshold shifts of hearing in healthy subjects. Int. Arch. Occup. Environ. Health 1997, 69, 433-436. [CrossRef]

37. Pekkarinen, J. Noise, impulse noise, and other physical factors: Combined effects on hearing. Occup. Med. 1995, 10, 545-559.

38. Ljungberg, J.; Neely, G.; Lundström, R. Cognitive performance and subjective experience during combined exposures to whole-body vibration and noise. Int. Arch. Occup. Environ. Health 2004, 77, 217-221. [CrossRef]

39. Arnberg, P.W.; Bennerhult, O.; Eberhardt, J.L. Sleep disturbances caused by vibrations from heavy road traffic. J. Acoust. Soc. Am. 1990, 88, 1486-1493. [CrossRef]

40. Goelzer, B.H.C.; Sehrndt, G. Occupational Exposure to Noise: Evaluation, Prevention and Control. Available online: https://www.who.int/occupational_health/publications/occupnoise/en/ (accessed on 19 January 2020).

41. Seidel, H. Selected health risks caused by long-term, whole-body vibration. Am. J. Ind. Med. 1993, 23, 589-604. [CrossRef]

42. Kim, J.-Y.; Shin, J.-S.; Lim, M.-S.; Choi, H.-G.; Kim, S.-K.; Kang, H.-T.; Koh, S.B.; Oh, S.S. Relationship between simultaneous exposure to ergonomic risk factors and work-related lower back pain: A cross-sectional study based on the fourth Korean working conditions survey. Ann. Occup. Environ. Med. 2018, 30, 58. [CrossRef]

43. Kim, S.; Jeong, W.; Kim, Y.K.; Jang, S.-I.; Park, E.-C. Gender Differences With Regard to Perceived Job Insecurity and Insomnia in a Working Population. J. Occup. Environ. Med. 2019, 61, e474-e479. [CrossRef] [PubMed]

44. American Psychiatric Association. Diagnostic and Statistical Manual of Mental Disorders: DSM-5; American Psychiatric Association: Arlington, TX, USA, 2013.

45. Jung, S.W.; Lee, J.-H.; Lee, K.-J.; Kim, H.-R. Association between Occupational Physicochemical Exposures and Headache/Eyestrain Symptoms among Korean Indoor/Outdoor Construction Workers. Saf. Health Work 2019, 10, 437-444. [CrossRef] [PubMed]

46. Boettcher, F.A.; Henderson, D.; Gratton, M.A.; Danielson, R.W.; Byrne, C.D. Synergistic Interactions of Noise and Other Ototraumatic Agents. Ear Hear. 1987, 8, 192-212. [CrossRef] [PubMed]

47. Sisto, R.; Botti, T.; Cerini, L.; Di Giovanni, R.; Marchetti, E.; Lunghi, A.; Sacco, F.; Sanjust, F.; Tirabasso, A.; Moleti, A. Synergistic effects of noise and hand-arm vibration on distortion product otoacoustic emissions in healthy subjects. Int. J. Ind. Ergon. 2017, 62, 48-54. [CrossRef]

48. Choi, S.B.; Yoon, J.-H.; Lee, W. The Modified International Standard Classification of Occupations defined by the clustering of occupational characteristics in the Korean Working Conditions Survey. Ind. Health 2019. [CrossRef]

(C) 2020 by the authors. Licensee MDPI, Basel, Switzerland. This article is an open access article distributed under the terms and conditions of the Creative Commons Attribution (CC BY) license (http://creativecommons.org/licenses/by/4.0/). 